\title{
PARAMETER OPTIMIZATION OF A VIRTUAL SYNCHRONOUS MACHINE IN A MICROGRID
}

\author{
Timo Dewenter, ${ }^{*}$ Wiebke Heins, ${ }^{* *}$ Benjamin Werther, ${ }^{* * *}$ Alexander K. Hartmann, ${ }^{*}$ Christian Bohn, ${ }^{* * * *}$ \\ and Hans-Peter Beck***
}

\begin{abstract}
Parameters of a virtual synchronous machine in a small microgrid are optimized. The dynamical behaviour of the system is simulated after a perturbation. The cost functional evaluates the system behaviour for different parameters. It is minimized by Parallel Tempering. Two perturbation scenarios are investigated. The resulting optimal parameters agree with analytical predictions. Dependent on the focus of the optimization, different optima are obtained for each scenario. During the transient the system leaves the allowed voltage and frequency bands only for a short time if the perturbation is within a certain range.
\end{abstract}

\section{Key Words}

Inverter-based microgrid, virtual synchronous machine, stochastic optimization, parallel tempering

\section{Introduction}

The number of renewable distributed energy resources (DERs) on low and medium voltage grid levels has increased in the last decades forced by political, ecological, and economical aspects. Many DERs are interfaced via inverters, which increase the need to find suitable control strategies and parameters for, e.g., frequency-power droop control in autonomous microgrids. Simulation methods, models and stability conditions for microgrids based on droop-controlled inverters are investigated in [1]-[7]. Stability analysis is done in [8], where conditions on the droop gains are derived. Simulations [9]-[12] have been used

* Institute for Physics, University of Oldenburg, Germany; e-mail: \{timo.dewenter, a.hartmann\}@uni-oldenburg.de

** Center for Industrial Mathematics, University of Bremen, Germany; e-mail: wiebke.heins@uni-bremen.de

*** Institute of Electrical Power Engineering and Energy Systems, Clausthal University of Technology, Germany; e-mail: benjamin.werther@tu-clausthal.de, beck@iee.tu-clausthal.de

**** Institute of Electrical Information Technology, Clausthal University of Technology, Germany; e-mail: bohn@iei. tu-clausthal.de

Recommended by Prof. G.O. Anderson

(DOI: 10.2316/Journal.203.2016.4.203-6270) to obtain optimal control parameters of inverters or distributed generators in microgrids. Particle swarm optimization is used in [13]-[19].

To enhance stability in microgrids, one can use programmable inverters with storage, as for example, the virtual synchronous machine (VISMA) [20]. It is a hysteresis-controlled three-phase inverter, whose setpoints are determined by a synchronous machine model implemented on a control computer. Inertia to improve transient stability of the grid is provided by a storage device. The VISMA is able to control (re-)active power bidirectionally and can be adjusted to meet specific power system requirements.

Here, the VISMA as grid-building element in a lowvoltage islanded microgrid with voltage source inverters is investigated. The basic control strategy is droop control [6], [8] for both voltage and frequency. We use the Parallel Tempering method [21], [22] for optimization of the VISMA parameters under varying transient loads (see e.g., [23]). Parallel Tempering allows to find (near-) optimal solutions for complex optimization problems [24], [25] efficiently. The objective of our analysis is to show that the optimization method is generally applicable to determine optimal control parameters in microgrids. Furthermore, different types of optima allow insights in the effects of the VISMA in combination with regular droop-controlled inverters in microgrids for the first time.

In Section 2, the simulation model is described. The optimization problem is stated in Section 3, and Section 4 explains the implementation. Results are presented in Section 5, and a conclusion is given in Section 6 .

\section{Microgrid Model}

\subsection{Lines and Loads}

Lines are modelled as algebraic equations describing the relation between voltage angles $\theta_{i}(t)$ and voltage magnitudes $V_{i}(t)$ at grid node $i \in[1, n]$ and (re-)active power flows [26]. Magnitudes and angles for all grid nodes are gathered in $V(t)=\left[V_{1}(t), V_{2}(t), \ldots, V_{n}(t)\right]^{\mathrm{T}}$ and $\theta(t)=$ $\left[\theta_{1}(t), \theta_{2}(t), \ldots, \theta_{n}(t)\right]^{\mathrm{T}}$. The (re-)active power injected at 


$$
\begin{aligned}
& P_{i}(V(t), \theta(t))=3\left(G_{i i} V_{i}^{2}(t)-\sum_{k \in N(i)} V_{i}(t) V_{k}(t)\left(G_{i k} \cos \left(\theta_{i}(t)-\theta_{k}(t)\right)+B_{i k} \sin \left(\theta_{i}(t)-\theta_{k}(t)\right)\right)\right) \\
& Q_{i}(V(t), \theta(t))=3\left(-B_{i i} V_{i}^{2}(t)-\sum_{k \in N(i)} V_{i}(t) V_{k}(t)\left(G_{i k} \sin \left(\theta_{i}(t)-\theta_{k}(t)\right)-B_{i k} \cos \left(\theta_{i}(t)-\theta_{k}(t)\right)\right)\right)
\end{aligned}
$$

Here, $G_{i i}=\hat{G}_{i i}+\sum_{k \in N(i)} G_{i k}, \quad B_{i i}=\hat{B}_{i i}+\sum_{k \in N(i)} B_{i k}$, $k \in N(i)$ denote summation over neighbours $k$ of node $i$, $\underline{\hat{Y}}_{i i}=\hat{G}_{i i}+\mathrm{j} \hat{B}_{i i}$ its shunt admittance, and $\underline{Y}_{i k}=G_{i k}+\mathrm{j} B_{i k}$ is the admittance of line $i k$.

The load is modelled as an external disturbance $S_{\text {load }}(t)=P_{\text {load }}(t)+\mathrm{j} Q_{\text {load }}(t)$. Algebraic constraints are introduced for the node $k$ to which the load is connected: $P_{\text {load }}(t)=P_{k}(V, \theta), Q_{\text {load }}(t)=Q_{k}(V, \theta)$.

\subsection{Droop-Controlled Inverters}

Following [8], inverters are modelled as controllable AC voltage sources described by differential equations. Each inverter is connected to the grid via an LCL-filter with inductance $L_{\mathrm{inv}}$ on the inverter side, filter capacitance $C_{\mathrm{f}}$, and coupling inductance $L_{\mathrm{C}}$. Here, $V_{i}(t)$ and $\theta_{i}(t)$ denote time-varying voltage magnitudes and angles over filter capacitances $C_{\mathrm{f}}$, assuming that these are controlled by the inverter.

Droop frequency and voltage control are based on decentralized proportional controllers. Its adaption to inverter-based microgrids has been investigated [2], [4]-[6], [27] extensively. Because droop control is only proportional, an offset error occurs if the system is permanently disturbed. The objective of the control strategy is that in steady state (denoted by $*$ ) of the closed loop system devices participating in droop control share the additional (re-)active power caused by the disturbance:

$$
\begin{aligned}
k_{\mathrm{P}, i}\left(P_{\mathrm{nom}, i}-P_{i}^{*}\left(V^{*}, \theta^{*}\right)\right) & =\omega_{i}^{*}-\omega_{\mathrm{nom}}, \\
k_{\mathrm{Q}, i}\left(Q_{\mathrm{nom}, i}-Q_{i}^{*}\left(V^{*}, \theta^{*}\right)\right) & =V_{i}^{*}-V_{\mathrm{nom}}
\end{aligned}
$$

Here, $P_{\text {nom }, i}$ and $Q_{\text {nom }, i}$ denote the nominal active and reactive power injections of each device. $V_{\text {nom }}$ and $\omega_{\text {nom }}$ denote the nominal voltage magnitude and frequency, respectively. The coefficients $k_{\mathrm{P}, i}$ and $k_{\mathrm{Q}, i}$ are parameters which determine the desired power sharing among devices. A common approach for the choice of droop coefficients $k_{\mathrm{P}, i}$ and $k_{\mathrm{Q}, i}$ is proportional load sharing (see [8] for analysis). Based on the power rating $S_{i}$ of each device and taking into account the legal limits for grid frequency and voltage magnitudes $(49.8-50.2 \mathrm{~Hz}$, and $207-253 \mathrm{~V}$, respectively), we obtain:

$k_{\mathrm{P}, i}=\frac{0.4 \cdot 2 \pi}{2 S_{i}} \frac{\mathrm{rad}}{\mathrm{s}}=\frac{0.4 \pi}{S_{i}} \frac{\mathrm{rad}}{\mathrm{s}}, \quad k_{\mathrm{Q}, i}=\frac{46 \mathrm{~V}}{2 S_{i}}=\frac{23 \mathrm{~V}}{S_{i}} \forall i$

In [8], voltage source inverters are modelled with instantaneous frequency $\dot{\theta}_{i}(t)=\omega_{\mathrm{sp}, i}(t)$, and first-order-delay voltage control $T_{\mathrm{inv}} \dot{V}_{i}(t)=-V_{i}(t)+V_{\mathrm{sp}, i}(t)$, where $\omega_{\mathrm{sp}, i}(t)$ and $V_{\mathrm{sp}, i}(t)$ denote freely adjustable frequency and voltage setpoints. Furthermore, power measurements are processed by low-pass filters with time constants $T_{i} \gg T_{\text {inv }}$. Choosing setpoints $\omega_{\mathrm{sp}, i}(t)$ and $V_{\mathrm{sp}, i}(t)$ according to (3) gives (see [8] for details):

$$
\begin{aligned}
\dot{\theta}_{i}(t) & =\omega_{i}(t) \\
T_{i} \dot{\omega}_{i}(t) & =-\omega_{i}(t)+\omega_{\mathrm{nom}}+k_{\mathrm{P}, i}\left(P_{\mathrm{nom}, i}-P_{i}(V(t), \theta(t))\right)
\end{aligned}
$$

$$
T_{i} \dot{V}_{i}(t)=-V_{i}(t)+V_{\mathrm{nom}}+k_{\mathrm{Q}, i}\left(Q_{\mathrm{nom}, i}-Q_{i}(V(t), \theta(t))\right)
$$

\subsection{VISMA}

The VISMA [20] is a programmable inverter which mimics the dynamics of a synchronous machine. It uses threephase grid voltages as input and three-phase currents as output. Its machine model, which defines how the programmable inverter is supposed to act, is adapted to fit in the overall model:

$$
\begin{aligned}
\dot{\theta}_{i}(t)= & \omega_{i}(t) \\
J \dot{\omega}_{i}(t)= & -\frac{k_{\mathrm{d}}}{T_{\mathrm{d}}} \omega_{i}(t)-\frac{k_{\mathrm{d}}}{T_{\mathrm{d}}} d(t) \\
& +\frac{1}{\omega_{i}(t)}\left(P_{\text {inject }}(t)-P_{i}(V(t), \theta(t))\right) \\
\dot{d}(t)= & -\frac{1}{T_{\mathrm{d}}} \omega_{i}(t)-\frac{1}{T_{\mathrm{d}}} d(t)
\end{aligned}
$$

Parameters are the virtual moment of inertia $J>0$, the mechanical damping factor $k_{\mathrm{d}}>0$, and the damping time constant $T_{\mathrm{d}}>0$. Compared to the VISMA model as stated in [28], this model was obtained by defining a "damping state" $d(t)=\frac{T_{\mathrm{d}}}{k_{\mathrm{d}}} M_{\mathrm{d}}(t)-\omega_{i}(t)$ and replacement of the momentum $M_{\text {mech }}(t)$ by $M_{\text {mech }}(t)=\frac{1}{\omega_{i}(t)} P_{\text {inject }}(t)$, with $P_{\text {inject }}(t)$ denoting the active power injected into the grid by the VISMA. In this setup, it is used for the purpose of droop and secondary frequency control, i.e., $P_{\text {inject }}(t)=P_{\text {droop }}(t)+P_{\text {secondary }}(t)$. According to (3), it is $P_{\text {droop }}(t)=P_{\text {nom }, i}+\frac{1}{k_{\mathrm{P}, i}}\left(\omega_{\text {nom }}-\omega_{i}(t)\right)$. Secondary frequency control is only performed by the VISMA and realized via an integral controller:

$$
\dot{x}(t)=K_{\mathrm{I}}\left(\omega_{\text {nom }}-\omega_{i}(t)\right), \quad P_{\text {secondary }}(t)=x(t)
$$

The voltage $E_{\mathrm{P}}[28]$ is represented here by the voltage magnitude $V_{i}(t)>0$ of the VISMA. Voltage dynamics of 


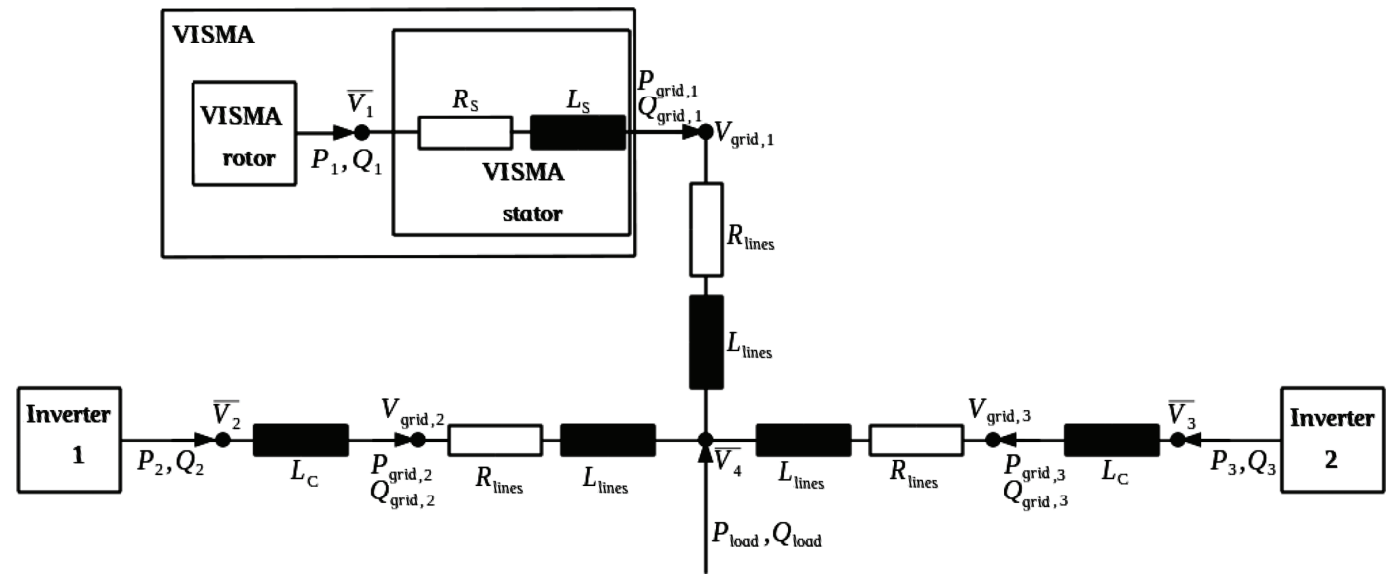

Figure 1. Scheme of the microgrid setup for simulation in perturbation scenarios.

the VISMA are assumed as a first-order delay with a fast time constant $T_{\mathrm{inv}, i}$. A specific voltage control strategy for the VISMA [29] is implemented using the root mean square value $V_{\text {grid }, i}(t)$ obtained from the grid voltage measurement between stator and grid (cf., Fig. 1) as:

$$
T_{\mathrm{inv}, i} \dot{V}_{i}(t)=-V_{i}(t)+V_{\text {nom }}+k_{\mathrm{V}}\left(V_{\text {nom }}-V_{\text {grid }, i}(t)\right)
$$

Furthermore, VISMA stator equations [28] are simplified as quasi-static and represented via an algebraic equation $\underline{Y}_{\mathrm{VISMA}}=\frac{1}{R_{\mathrm{S}}+\mathrm{j} \omega_{\mathrm{nom}} L_{\mathrm{S}}}$ with stator resistance $R_{\mathrm{S}}>0$ and stator inductance $L_{\mathrm{S}}>0$.

\subsection{Overall Simulation Model}

We choose the VISMA node (node 1) as reference node. All voltage angles are replaced by their difference to the reference node's voltage angle via $\Delta \theta_{i}(t):=\theta_{i}(t)-\theta_{1}(t) \forall i$. Naturally, we have $\Delta \theta_{1}(t) \equiv 0$ and therefore the state $\Delta \theta_{1}(t)$ and (8) are not needed to describe the full system. A new vector is defined for angle states as $\Delta \theta(t)=\left[\Delta \theta_{2}(t), \ldots, \Delta \theta_{n}(t)\right]^{\mathrm{T}} \in \mathbb{R}^{n-1} . \quad$ For lines and loads, $\theta_{i}(t)$ can be directly replaced by $\Delta \theta_{i}(t) \forall i$. For the inverters, assuming that none of them is connected to node $1,(5)$ is replaced by $\Delta \dot{\theta}_{i}(t)=\omega_{i}(t)-\omega_{1}(t)$. Given the complex power $\underline{S}_{i}=P_{i}+\mathrm{j} Q_{i}$, complex coupling admittance $\underline{Y}_{\text {coupl }}=\frac{1}{R_{\mathrm{S}}+\mathrm{j} \omega_{\text {nom }} L_{\mathrm{S}}}$ (or, for the inverters $\left.\underline{Y}_{\text {coupl }}=\frac{1}{\mathrm{j} \omega_{\text {nom }} L_{\mathrm{C}}}\right)$, and complex voltage $\underline{V}_{i}=V_{i} \mathrm{e}^{\mathrm{j} \Delta \theta_{i}}$, we obtain the complex voltage $\underline{V}_{\text {grid }, i}=V_{\text {grid }, i} \mathrm{e}^{\mathrm{j} \Delta \theta_{\text {grid }, i}}$ between VISMA stator or inverter filters and grid as $\underline{V}_{\mathrm{grid}, i}(t)=\frac{\left|\underline{\underline{V}}_{i}(t)\right|^{2}}{\overline{\underline{V}}_{i}(t)}-\frac{\overline{\underline{S}}_{i}(t)}{3 \underline{\underline{\underline{Y}}}_{\mathrm{coupl}} \overline{\underline{\underline{V}}}_{i}(t)}$.

\section{Problem Statement}

\subsection{Optimization Constraints}

The objective of the optimization is to find parameters $J$, $k_{\mathrm{d}}, T_{\mathrm{d}}$, and $K_{\mathrm{I}}$ for the VISMA which positively influence the overall system behaviour after a perturbation. To avoid undesired or physically impossible behaviour, optimization variables have to be bounded by user-defined constraints.
The first constraint assures that the VISMA does not react faster than the other inverters. Therefore, we investigate the dynamics of the VISMA (cf., (9) and (10)). For the purpose of deriving a simple model as reference for the optimization constraints, the following simplifications are used: only the machine model is investigated, grid and stator equations, differential equations of voltage dynamics, and secondary control are not considered. Taking $P_{1}(V(t), \Delta \theta(t))$ as system input $u(t)$, linearizing around the equilibrium point $u^{*}=P_{\text {nom }, 1}, \omega_{1}^{*}=\omega_{\text {nom }}$ and $d^{*}=-\omega_{\text {nom }}$, and applying the Laplace transform gives the transfer function:

$$
\begin{aligned}
G_{\mathrm{VISMA}, \operatorname{lin}}(s) & =-\frac{k_{\mathrm{P}, 1}\left(T_{\mathrm{d}} s+1\right)}{\frac{1}{\Omega^{2}} s^{2}+\frac{D}{2 \Omega} s+1}, \quad c:=\frac{1}{k_{\mathrm{P}, 1} \omega_{\mathrm{nom}}}, \\
D & :=\frac{\frac{1}{c}\left(k_{\mathrm{d}}+J\right)+T_{\mathrm{d}}}{2 \sqrt{\frac{1}{c} J T_{\mathrm{d}}}}, \quad \Omega:=\frac{1}{\sqrt{\frac{1}{c} J T_{\mathrm{d}}}}
\end{aligned}
$$

The poles are real because $D>1$ for any choice of parameters (proof omitted), therefore:

$$
\begin{aligned}
& s_{\text {pole }, 1}=-\Omega\left(D+\sqrt{D^{2}-1}\right) \\
& s_{\text {pole }, 2}=-\Omega\left(D-\sqrt{D^{2}-1}\right)
\end{aligned}
$$

From linear system theory, it is known that $\tau_{1 / 2}=$ $-\frac{1}{s_{\text {pole }, 1 / 2}}$ determines the exponential decay rate. This results in the constraint $\max _{i}\left(T_{i}\right) \leq \min \left(\tau_{1}, \tau_{2}\right)$, where $T_{i}$ is the time constant of the regular inverters. Assuming stable system configurations, i.e., $s_{\text {pole }, 1}, s_{\text {pole }, 2}<0$, we conclude that $\tau_{1}<\tau_{2}$, and therefore:

$$
\max _{i}\left(T_{i}\right) \leq \frac{1}{\Omega\left(D+\sqrt{D^{2}-1}\right)}
$$

A second constraint defines an upper bound for the parameter $K_{\mathrm{I}}$ of the integral controller (11) based on the design preference that integral control action should occur only after the first part of the transient caused by droop control is finished. For the simplified model (13), more 
than $95 \%$ of the absolute value of the step size are reached after $3 \max _{i=1,2}\left(\tau_{i}\right)$ because of the exponential character of the linearized system's step response. The response time of the integral controller should be larger. A lower bound for the response time $\frac{1}{K_{\mathrm{I}}}$ of the integral controller, by using that $x(t)$ is scaled by $\frac{1}{J \omega_{\text {nom }}}$ in $(13)$, is given by:

$$
K_{\mathrm{I}} \leq \frac{J \omega_{\mathrm{nom}}}{3 \tau_{2}}=\frac{1}{3} J \omega_{\mathrm{nom}} \Omega\left(D-\sqrt{D^{2}-1}\right)
$$

\subsection{Cost Functional}

The cost $E$ to be optimized contains three parts and parameters $\alpha>0$ and $\beta>0$ :

$$
E\left[\Delta f, \Delta V, \delta_{\mathrm{f}}, \delta_{\mathrm{V}}\right]=t_{\text {final }}+\alpha \cdot\left(k_{\mathrm{d}}+J\right)+\underbrace{\left(\Delta f / \delta_{\mathrm{f}}+\Delta V / \delta_{\mathrm{V}}\right)}_{=: \Sigma} / \beta
$$

As $\Sigma$ depends on system trajectories, $E$ is a functional. Parameters $\alpha$ and $\beta$ allow to shift the focus of the optimization. First, the transient after a perturbation should be short, i.e., $t_{\text {relax }} \rightarrow \min$. Time $t_{\text {relax }}$ is the relaxation time of the system after a perturbation, defined here as the latest time instant in which the frequencies reach $49.999 \mathrm{~Hz}$ again. Then, $t_{\text {final }}=t_{\text {relax }}-t_{0}$, where $t_{0}$ is the moment of the jump in load. Second, the peak depth in the transients of frequency and voltage should be small to avoid damage on electronic devices, i.e., $\Delta f / \delta_{\mathrm{f}}+\Delta V / \delta_{\mathrm{V}} \rightarrow$ min, where $\Delta f=\max _{\left.\{i \in\{1,2,3\}\}, t>t_{0}\right\}}\left|f_{i}(t)-f_{i}\left(t_{0}\right)\right|$ is the maximum frequency deviation, $f_{i}$ being the frequency at node $i$. The maximum voltage deviation is $\Delta V=\max _{\left\{i \in\{1,2,3,4\}, t>t_{0}\right\}}$ $\left|V_{\text {grid }, i}(t)-V_{\text {grid }, i}\left(t_{0}\right)\right|$, with grid voltage $V_{\text {grid }, i}(t)$.

Third, a trade-off must be found between the required storage capacity of the VISMA, which should be as small as possible, and the energy that is used to keep up its virtual inertia. By setting $M_{\text {mech }}=0$ and integrating (9) over time, the energy provided to or taken from the microgrid by the VISMA is:

$$
\begin{aligned}
E_{\mathrm{VISMA}}= & -\frac{1}{2}\left(J+k_{\mathrm{d}}\right)\left(\omega^{2}\left(t_{2}\right)-\omega^{2}\left(t_{1}\right)\right) \\
& +T_{\mathrm{d}} \int_{t_{1}}^{t_{2}} \omega_{i}(t) \dot{M}_{\mathrm{d}}(t) \mathrm{d} t
\end{aligned}
$$

where $T_{\mathrm{d}}$ is responsible for scaling the energy loss due to damping. The other part of the energy is dominated by $J+k_{\mathrm{d}}$. To avoid unnecessary large storage capacities, $k_{\mathrm{d}}+J$ is minimized. The constraints of the optimization problem allow some insights into the structure of the optima in advance. Choosing a very small $k_{\mathrm{d}}$ and $T_{\mathrm{d}}$ close to $\max _{i=1,2} T_{i}$ gives values as close as possible to the lower bound of (15). On the one hand, this indicates that an optimization with focus on keeping the transient behaviour of the VISMA close to those of regular inverters (i.e., minimizing the virtual inertia $J+k_{\mathrm{d}}$ and the transient time $\left.t_{\text {final }}\right)$ will give results close to $T_{\mathrm{d}} \approx \max _{i=1,2} T_{i}, k_{\mathrm{d}} \approx 0$ and $J \approx c \max _{i=1,2} T_{i}$. Minimizing mainly $t_{\text {final }}$ on the other hand leads to a large value within limits given by (16), namely $K_{\mathrm{I}} \approx \frac{1}{3 k_{\mathrm{P}, 1}}$.

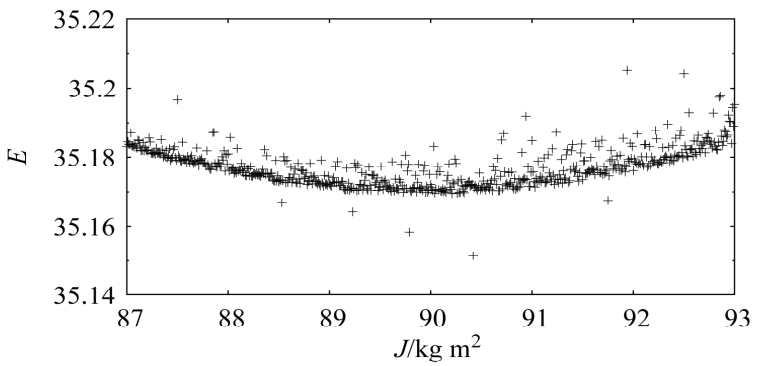

Figure 2. Two-dimensional projection of the energy landscape (value of cost functional $E(17)$ ) for scenario 1, close to min. \#2. The parameters $T_{\mathrm{d}}=0.6, k_{\mathrm{d}}=2.6 \times 10^{-4}$, and $K_{\mathrm{I}}=1,060$ are fixed, $J$ is varied.

\section{Implementation}

The energy landscape (see Fig. 2) is rough with many local minima, in particular due to a stochastic term needed for the initial conditions when solving the differential equations, preventing the application of standard, e.g., gradient-based, methods. Instead, Parallel Tempering is used, which works for harder optimization problems, but is easy to implement.

\subsection{Parallel Tempering}

The optimization algorithm works as follows. The configurations of the system are sampled according to the Boltzmann probability distribution $P\left(E_{i}\right)=1 / Z \exp \left(-E_{i} / \Theta\right)$, where $Z$ is a normalization constant, $E_{i}$ is the energy of configuration $i$, and $\Theta$ an artificial temperature. This is achieved via a special Monte Carlo (MC) sampling, the Metropolis algorithm [30], where in each iteration, a new candidate configuration with corresponding energy $E_{2}$ is created and accepted with probability:

$$
p_{\mathrm{Metr}}=\min \left\{1, e^{-\left(E_{2}-E_{1}\right) / \Theta}\right\}
$$

where $E_{1}$ is the energy of the current state. As (19) fulfils detailed balance, sampling according to a Boltzmann distribution is ensured. From physics we know that for $\Theta \rightarrow 0$, the energy obtains a minimum $E \rightarrow E_{\text {min }}$. This leads to the idea of Simulated Annealing [31], where the temperature of an $\mathrm{MC}$ simulation is gradually decreased until a minimum is found. This approach can get stuck in a local minimum. An improvement is to simulate the system at various temperatures $\Theta_{i}$. This can be done by Parallel Tempering [21], [22], where a random walk in temperature space is performed. To preserve detailed balance and equilibrium for an infinite number of iterations, the Metropolis criterion [21] with energies $E(\cdot)$ is used:

$p_{\text {Swap }}=\min \left\{1, \exp \left(\left[\frac{1}{\Theta_{k}}-\frac{1}{\Theta_{k+1}}\right]\left[E\left(y_{k}\right)-E\left(y_{k+1}\right)\right]\right)\right\}$

Two neighbouring configurations with temperatures $\Theta_{k}, \Theta_{k+1}(k \in[1, n-1])$ can be exchanged. In each such swap, $k \in\{1,2,3, \ldots, n-1\}$ is chosen at random with equal probability. 
Table 1

Parameters Used for the Optimization in All Scenarios. Values for $T_{2 / 3}$ Taken from [8]

\begin{tabular}{|l|c|c|c|c|c|c|c|c|}
\hline$L_{\text {lines }}$ & $L_{\mathrm{S}}$ & $R_{\text {lines }}$ & $R_{\mathrm{S}}$ & $L_{\mathrm{C}}$ & $T_{1}$ & $T_{2 / 3}$ & $k_{\mathrm{V}}$ & $Q_{\text {nom }, 1 / 2 / 3}$ \\
\hline $1.514 \mathrm{mH}$ & $42.0 \mathrm{mH}$ & $0.0 \Omega$ & $0.3 \Omega$ & $1.8 \mathrm{mH}$ & $0.01 \mathrm{~s}$ & $0.5 \mathrm{~s}$ & $10.0 \mathrm{~V} / \mathrm{V}$ & $0.0 \mathrm{var}$ \\
\hline
\end{tabular}

Table 2

Parameters Used for the Optimization in Scenario 1

\begin{tabular}{|l|c|c|c|c|c|}
\hline$S_{1 / 2 / 3}$ & $P_{\text {nom }, 1 / 2 / 3}$ & $k_{\mathrm{P}, 1 / 2 / 3}$ & $k_{\mathrm{Q}, 2 / 3}$ & $P_{\text {load }}$ before Jump & $P_{\text {load }}$ after Jump \\
\hline $4,000.0 \mathrm{VA}$ & $500.0 \mathrm{~W}$ & $3.1416 \times 10^{-4} \mathrm{rad} /(\mathrm{s} \mathrm{VA})$ & $5.75 \cdot 10^{-3} \mathrm{~V} / \mathrm{VA}$ & $1,500.0 \mathrm{~W}$ & $4,500.0 \mathrm{~W}$ \\
\hline
\end{tabular}

Table 3

Optimal Parameter Sets for Scenario $1\left(R_{\text {perc }}=0.4\right.$, Focus on Frequency Peak: $\left.\delta_{\mathrm{f}}=0.05, \delta_{\mathrm{V}}=10^{40}\right)$. Errors of $E$ Resulting from 50 Runs with Different Initial Conditions

\begin{tabular}{|c|c|r|c|c|c|c|c|r|c|c|c|c|}
\hline$\#$ & $J$ & $k_{\mathrm{d}} / 10^{-4}$ & $T_{\mathrm{d}}$ & $K_{\mathrm{I}}$ & $E$ & $\alpha$ & $\beta$ & $J+k_{\mathrm{d}}$ & $\Sigma$ & $t_{\text {final }}$ & $\alpha\left(J+k_{\mathrm{d}}\right)$ & $\Sigma / \beta$ \\
\hline 1 & 5.0895 & 1.1857 & 0.5029 & $1,054.56$ & $108.93(6)$ & 7 & 0.027 & 5.090 & 0.994 & 36.483 & 35.627 & 36.820 \\
\hline 2 & 91.479 & 2.5800 & 0.5917 & $1,060.97$ & $35.12(2)$ & 0.07 & 2.7 & 91.480 & 0.817 & 28.415 & 6.4036 & 0.3026 \\
\hline 3 & 5.0692 & 1.0071 & 0.5163 & 975.67 & $3,624.89(9)$ & 700 & 0.027 & 5.069 & 1.000 & 39.379 & $3,548.494$ & 37.026 \\
\hline 4 & 50.894 & 10.1498 & 1.2539 & $1,053.54$ & $3,425(46)$ & 7 & $2.7 \times 10^{-4}$ & 50.895 & 0.820 & 32.913 & 356.265 & $3,036.54$ \\
\hline
\end{tabular}

\subsection{Optimization Algorithm}

Within an optimization procedure, one of two perturbation scenarios is considered. Both are based on a step in load. During the transient it is checked whether the usual frequency and voltage ranges are met (see (4)). If these are not fulfilled, the parameter set is rejected, i.e., $E=\infty$. Before (17) is calculated in the simulation, the constraints (15) and (16) are checked. If they are not fulfilled, the parameter set is rejected.

We use from the GNU scientific library (GSL) [32]: a hybrid method (Newton and dogleg step) for solving the steady-state equations, where the results are used as initial conditions for the Runge-Kutta-Fehlberg method to solve the differential equations. For parallelization, we use OpenMPI [33]. The 12 temperatures used in the simulations are $\Theta_{i} \in\left\{0.01,0.02,0.07,0.2,0.5,1,3,7,20,50,100,10^{9}\right\}$, where $10^{9}$ corresponds to the acceptance of every new state except the ones that violate $(15),(16)$ or lead to an unstable system state. For each temperature $\Theta_{i}$, the MC sampling is performed in the following way:

1. Calculate value of cost functional $E_{1}$ with given parameter set $\Phi=\left(J, k_{\mathrm{d}}, T_{\mathrm{d}}, K_{\mathrm{I}}\right)$.

2. Choose one parameter $O$ of the four parameters from $\Phi$ with equal probability at random.

3. Calculate $O^{\prime}=O \cdot m$ with $m=\left|1+R_{\text {perc }} \cdot r\right|$, where $r \in[-1,1]$ is a random number.

4. Calculate new value of $E_{2}$ with modified parameter set $\Phi^{\prime}$ in which $O$ has been replaced by $O^{\prime}$.

5. Accept the new parameter set $\Phi^{\prime}$ with Metropolis probability (19).

The steps $2-5$ are repeated $N_{\text {params }} \cdot 2=4 \cdot 2=8$ times. After two such sweeps are performed for each temperature, $n-1$ swap attempts are done. For each of these attempts, the procedure is the following: First, choose a configuration $k \in[1, n-1]$ uniformly at random. Then, exchange the two configurations $y_{k}$ and $y_{k+1}$ with the swap probability given by (20). For each parameter set $(\alpha, \beta), 200$ swaps are performed with $R_{\text {perc }}=0.8$. The minimum of $E$ is found by taking the minimum of all temperatures $\Theta_{i}$ leading to $\Phi_{\text {min }}$. Another simulation [34] with $\Phi_{\min }$ as initial parameter set is started with 200 swaps and $R_{\text {perc }}=0.4$.

\section{Results}

\subsection{Optimization Results}

A microgrid in a radial topology in island mode is considered for different perturbation scenarios, see Fig. 1. We neglect ohmic grid losses and focus on the frequency peak, i.e., $\delta_{\mathrm{V}}=10^{40}$. The perturbation is a jump in active load. Table 1 shows the parameters which remain the same for all scenarios.

\subsubsection{Scenario 1}

In this scenario, equal nominal and rated powers for all devices are assumed and a step decrease of the load power of $3 \mathrm{~kW}$ is done. See Table 2 for all parameters. In the last three columns of Table 3 the values of the parts of the cost functional (17) are given, which reflect on which part of the functional the optimization has been focused. For the first minimum (\#1) the three parts of (17) have the same weight, the second minimum (min.) focuses on $t_{\text {final }}$, the third on the value of $J+k_{\mathrm{d}}$, and the fourth on small frequency peaks $(\Sigma)$. For the second, third and fourth min. the focus is reflected in the result, i.e., min. \#2 has 

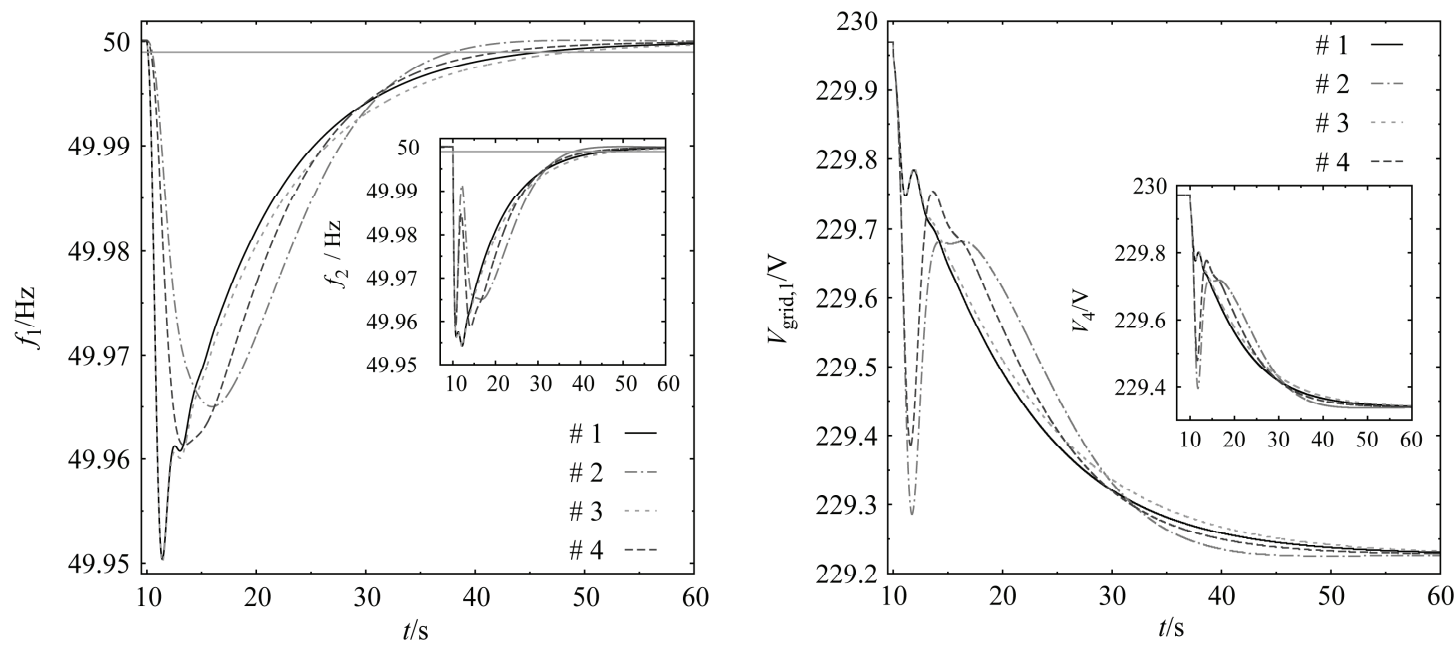

Figure 3. Comparison of the minima for scenario 1 (see Table 3) for frequencies (left) and voltages (right).
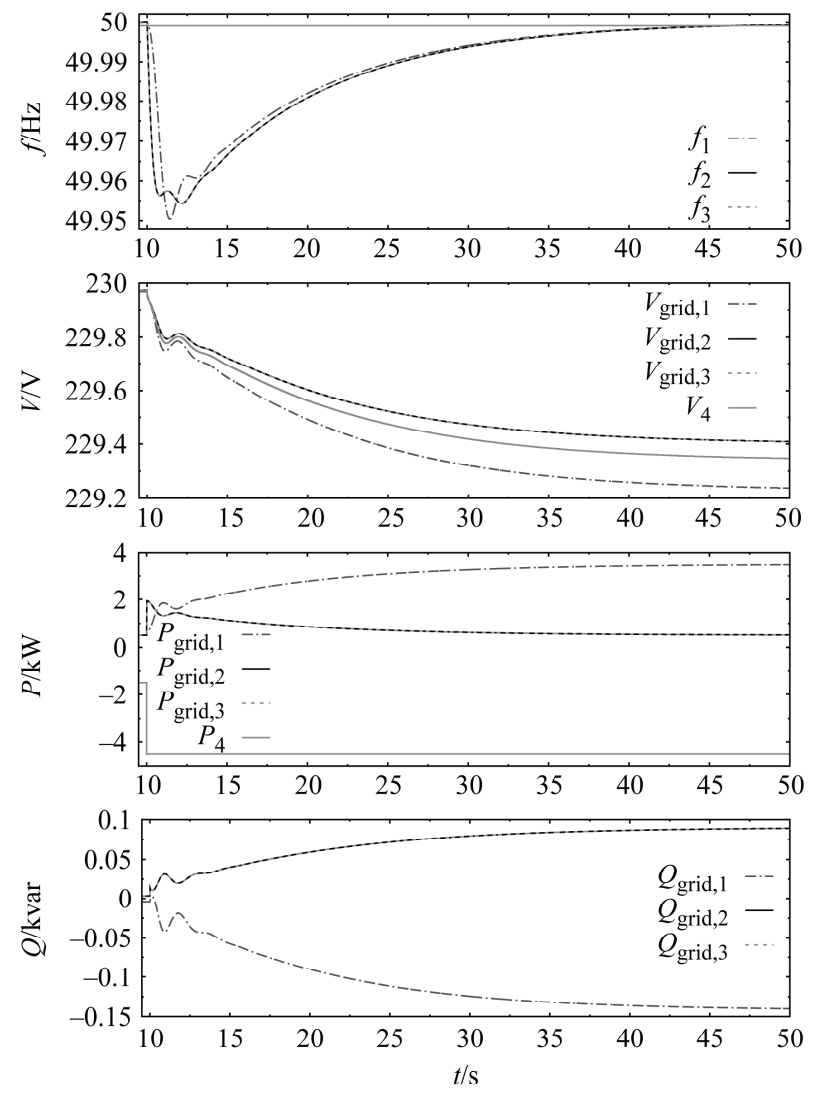

Figure 4. Minimum \#1 of Scenario 1. the smallest value of $t_{\text {final }}$, min. \#3 the smallest of $J+k_{\mathrm{d}}$. Min. \#4 gives a comparably small value for $\Sigma$, but only a local min. was found, as in min. \#2 it is even smaller.

In Section 3.2, it was stated that $T_{\mathrm{d}} \approx \max _{i=1,2} T_{i}=0.5$, $k_{\mathrm{d}} \approx 10^{-4}$ (because it is bounded by this value) and $J \approx c \max _{i=1,2} T_{i} \approx 10.13 \cdot 0.5 \approx 5.07$ in cases where $J+k_{\mathrm{d}}$ and $t_{\text {final }}$ are minimized with equal weighting, and $K_{\mathrm{I}} \approx \frac{1}{3 k_{\mathrm{p}, 1}} \approx 1,061.03$, if the minimization focuses on $t_{\text {final }}$. These values are close to min. \#1 and min. \#3, and min. \#2 for focussing on $t_{\text {final }}$. Weightings on other parts of the cost functional (e.g., min. \#4) lead to minima which are further away from the bounds of the optimization constraints.

The results from Table 3 are visualized in Fig. 3 by a comparison for VISMA frequencies and voltages for all four minima. The remaining grid values are shown only for the first minimum in Fig. 4.

\subsubsection{Scenario 2}

In this scenario, we assume different rated and nominal active powers and a step in load of $7 \mathrm{~kW}$ (see Table 4). The optimal parameter sets obtained for this setup are shown in Table 5 . Figure 5 shows the system behaviour for min. \#1 of Scenario 2. The VISMA's reaction is very slow due to its high virtual inertia (for min. \#1, $J \approx 11.5$ ), therefore the inverters balance the sudden power demand directly after the load jump and provide active power at a value $(\sim 8 \mathrm{~kW})$ above their nominal rated power values $S_{2 / 3}$. Inverters for island grids usually allow this for a short time.

Table 4

Parameters Used for the Optimization in Scenario 2

\begin{tabular}{|c|c|c|c|c|c|c|c|c|c|}
\hline$S_{1}$ & $S_{2}$ & $S_{3}$ & $P_{\text {nom }, 1}$ & $P_{\mathrm{nom}, 2}$ & $P_{\text {nom }, 3}$ & \multicolumn{2}{|c|}{$k_{\mathrm{P}, 1}$} & \multicolumn{2}{|r|}{$k_{\mathrm{P}, 2}$} \\
\hline $9.0 \mathrm{kVA}$ & $3.0 \mathrm{kVA}$ & $1.0 \mathrm{kVA}$ & $1.0 \mathrm{~kW}$ & $1.5 \mathrm{~kW}$ & $0.5 \mathrm{~kW}$ & \multicolumn{2}{|c|}{$1.3963 \times 10^{-4} \mathrm{rad} /(\mathrm{s} \mathrm{VA})$} & 4.1888 & $\times 10^{-4} \mathrm{ra}$ \\
\hline \multicolumn{3}{|c|}{$k_{\mathrm{P}, 3}$} & \multicolumn{2}{|c|}{$k_{\mathrm{Q}, 2}$} & \multicolumn{2}{|r|}{$k_{\mathrm{Q}, 3}$} & \multicolumn{2}{|c|}{$P_{\text {load }}$ before Jump } & $P_{\text {load }}$ after Jump \\
\hline \multicolumn{3}{|c|}{$12.5664 \times 10^{-4} \mathrm{rad} /(\mathrm{s} \mathrm{VA})$} & \multicolumn{2}{|c|}{$7.67 \times 10^{-3} \mathrm{~V} / \mathrm{VA}$} & \multicolumn{2}{|c|}{$23.0 \times 10^{-3} \mathrm{~V} / \mathrm{VA}$} & \multicolumn{2}{|l|}{$3.0 \mathrm{~kW}$} & $10.0 \mathrm{~kW}$ \\
\hline
\end{tabular}


Table 5

Optimal Parameter Sets for Scenario $2\left(R_{\text {perc }}=0.4\right.$, Focus on Frequency Peak: $\left.\delta_{\mathrm{f}}=0.2, \delta_{\mathrm{V}}=10^{40}\right)$. Errors of $E$ Resulting from 50 Runs with Different Initial Conditions.

\begin{tabular}{|c|c|c|c|c|c|c|c|c|c|c|c|c|}
\hline$\#$ & $J$ & $k_{\mathrm{d}} / 10^{-4}$ & $T_{\mathrm{d}}$ & $K_{\mathrm{I}}$ & $E$ & $\alpha$ & $\beta$ & $J+k_{\mathrm{d}}$ & $\Sigma$ & $t_{\text {final }}$ & $\alpha\left(J+k_{\mathrm{d}}\right)$ & $\Sigma / \beta$ \\
\hline 1 & 11.4986 & 1.1595 & 0.5035 & $2,379.26$ & $59.26(1)$ & 1.7 & 0.045 & 11.499 & 0.902 & 19.671 & 19.548 & 20.046 \\
\hline 2 & 59.3043 & 4.2760 & 0.9356 & $2,387.04$ & $14.99(1)$ & 0.017 & 4.5 & 59.305 & 0.887 & 13.781 & 1.0082 & 0.1971 \\
\hline 3 & 11.4076 & 1.0139 & 0.5064 & $2,348.85$ & $1,979.32(1)$ & 170 & 0.045 & 11.408 & 0.902 & 19.967 & $1,939.309$ & 20.040 \\
\hline 4 & 16.8974 & 13.2759 & 0.6524 & $2,382.76$ & $2,039.5(5)$ & 1.7 & $4.5 \times 10^{-4}$ & 16.899 & 0.896 & 19.076 & 28.728 & $1,991.7$ \\
\hline
\end{tabular}
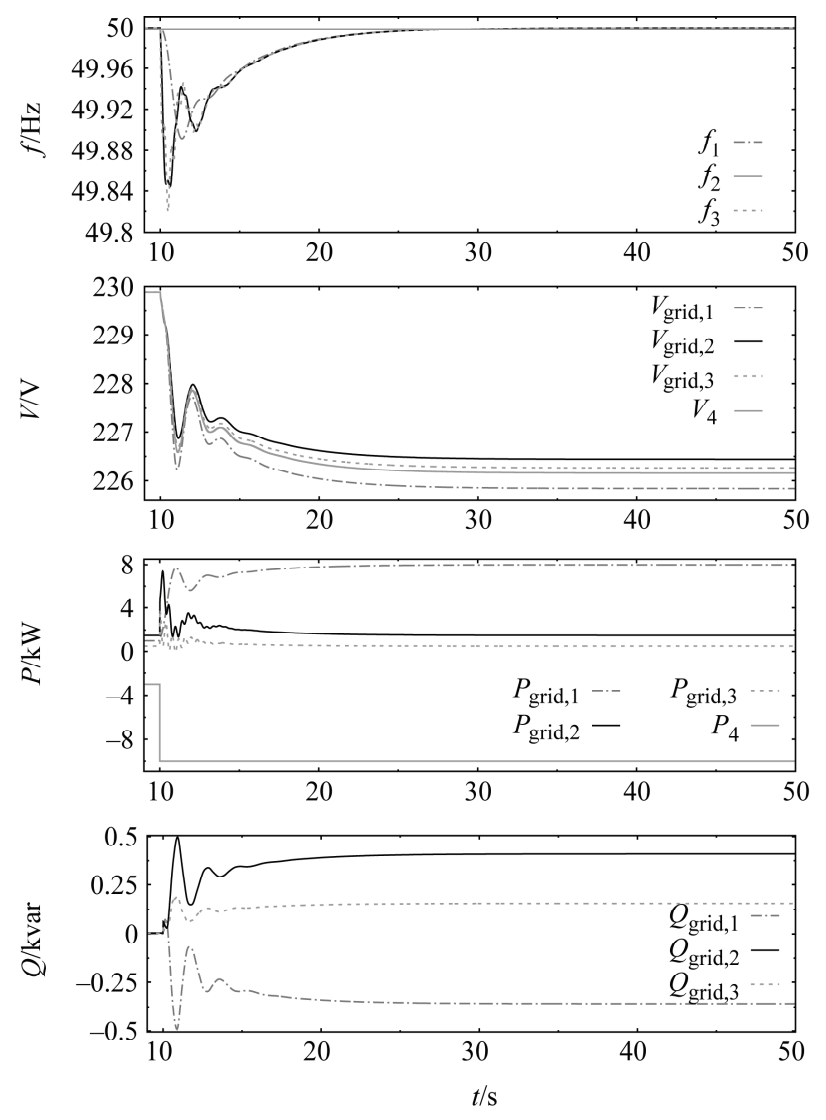

Figure 5. Minimum \#1 of Scenario 2.

Despite the different system behaviour compared to Scenario 1, the effect of the optimization with different weights is clearly reflected in the values of the cost functional (see Table 5). The very small $t_{\text {final }}$ for min. \#2 is due to an overshoot in frequencies (figure not shown): after reaching $49.999 \mathrm{~Hz}$ for the first time, $f_{1}$ leaves the range of $[49.999,50.001]$ again before returning to $50 \mathrm{~Hz}$. Optimizations for the same scenarios with ohmic losses in lines have been performed (results not shown). Though transients show different characteristics, almost the same parameter sets are found.

\section{Conclusion}

Parameters of a VISMA in an islanded microgrid with radial topology containing two inverters and a load have been optimized using Parallel Tempering. By varying additional parameters in the cost functional, the focus of the optimization was shifted. For two perturbation scenarios, minima of the cost functional were found which are stable solutions within the prescribed boundaries. The results show that this optimization procedure is in general applicable to the task of parameter optimization in a microgrid. It is also shown that through the proper setting of the VISMA's parameters its functionality can be adapted to different participants in the grid. The values obtained by analytical investigation in Section 3.2 seem to offer a "rule of thumb" for a good parameter region. However, the effects of each parameter in complex situations are not obvious. For other topologies, devices or disturbances, totally different parameter sets might be needed. To find a good parameter set for a given microgrid setup, various disturbances should be analyzed.

In future research, other forms of the constraints for the VISMA parameters and cost functionals should be investigated. Larger microgrids with other power generating systems can be put under scrutiny. The proposed approach could be transferred to the optimization of parameters in other control strategies. An extension would be the study of different disturbance scenarios, where one uses, for example, a series of steps in load. Finally, theoretical results should be confirmed by measurements in an appropriate laboratory.

\section{Acknowledgement}

Simulations were performed at the HERO cluster of the Carl von Ossietzky Universität Oldenburg, funded by the DFG through its Major Research Instrumentation Programme (INST 184/108-1 FUGG) and the Ministry of Science and Culture of the Lower Saxony State. Financial support was obtained by the Lower Saxony research network "Smart Nord" which acknowledges the support of the Lower Saxony Ministry of Science and Culture through the "Niedersächsisches Vorab" grant programme (grant ZN2764).

\section{References}

[1] N. Pogaku, M. Prodanovic, and T. Green, Modeling, analysis, and testing of autonomous operation of an inverter-based microgrid, IEEE Transactions on Power Electronics, 22, 2007, 613. 
[2] E. Coelho, P. Cortizo, and P. Garcia, Small signal stability for parallel connected inverters in stand-alone AC supply systems, IEEE Transactions on Industry Applications, 38, 2002, 533.

[3] M. Marwali, J. Jung, and A. Keyhani, Stability analysis of load sharing control for distributed generation systems, IEEE Transactions on Energy Conversion, 22, 2007, 737.

[4] E. Barklund, N. Pogaku, M. Prodanovic, C. HernandezAramburo, and T.C. Green, Energy management in autonomous microgrid using stability-constrained droop control of inverters, IEEE Transactions on Power Electronics, 23, $2008,2346$.

[5] N.L. Soultanis, S.A. Papathanasiou, and N.D. Hatziargyriou, A stability algorithm for the dynamic analysis of inverter dominated unbalanced LV microgrids, IEEE Transactions on Power Systems, 22(1), 2007, 294-304.

[6] A. Engler, Applicability of droops in low voltage grids, DER Journal, 1(1), 2005, 3-15.

[7] J.W. Simpson-Porco, F. Dörfler, and F. Bullo, Synchronization and power sharing for droop-controlled inverters in islanded microgrids, Automatica, 49(9), 2013, 2603-2611.

[8] J. Schiffer, R. Ortega, A. Astolfi, J. Raisch, and T. Sezi, Conditions for stability of droop-controlled inverter-based microgrids, Automatica, 50, 2014, 2457-2469.

[9] A. Raghami, M.T. Ameli, and M. Hamzeh, Online droop tuning of a multi-DG microgrid using cuckoo search algorithm, Electric Power Components and Systems, 43(14), 2015, 1583-1595.

[10] R.B. Godoy, J.O.P. Pinto, C.A. Canesin, E.A.A. Coelho, and A.M.A.C. Pinto, Differential-evolution-based optimization of the dynamic response for parallel operation of inverters with no controller interconnection, IEEE Transactions on Industrial Electronics, 59, 2012, 2859.

[11] S. Mishra, G. Mallesham, and A. Jha, Design of controller and communication for frequency regulation of a smart microgrid, IET Renewable Power Generation, 6, 2011, 248.

[12] M.J. Sanjari and G.B. Gharehpetian, Game-theoretic approach to cooperative control of distributed energy resources in islanded microgrid considering voltage and frequency stability, Neural Computing and Applications, 25, 2013, 343.

[13] I.-Y. Chung, W. Liu, D.A. Cartes, and S.-I. Moon, Control parameter optimization for multiple distributed generators in a microgrid using particle swarm optimization, European Transactions on Electrical Power, 21, 2011, 1200.

[14] M. Hassan and M. Abdio, Optimal design of microgrids in autonomous and grid-connected modes using particle swarm optimization, IEEE Transactions on Power Electronics, 26, 2011, 755

[15] W. Al-Saedi, S. Lachowicz, D. Habibi, and O. Bass, Power quality enhancement in autonomous microgrid operation using particle swarm optimization, International Journal of Electrical Power \& Energy Systems, 42, 2012, 139.

[16] H. Bevrani, F. Habibi, P. Babahajyani, M. Watanabe, and Y. Mitani, Intelligent frequency control in an AC microgrid: Online PSO-based fuzzy tuning approach, IEEE Transactions on Smart Grid, 3, 2012, 1935.

[17] L. Yu, M. Chen, D.C. Yu, L. Zhang, F. Yang, and J. Zhai, A novel information exchange particle swarm optimization for microgrid multi-objective dynamic optimization control, Journal of Renewable and Sustainable Energy, 6(2), 2014, 023114 .

[18] Y. Zeng and S. Yanguang, Enhanced multi-objective particle swarm optimization for optimal reactive power dispatch considering voltage stability, International Journal of Power and Energy Systems, 34, 2014, 116-24.

[19] P. Wang, J. Wan, R. Luo, F. Zhao, and X. Wei, Control parameter optimization for AP1000 reactor using particle swarm optimization, Annals of Nuclear Energy, 87, Part 2, 2016, 687-695.

[20] H.-P. Beck and R. Hesse, Virtual synchronous machine, IEEE Ninth International Conf. on Electrical Power Quality and Utilisation (EPQU), Barcelona, Spain, 2007.

[21] K. Hukushima and K. Nemoto, Exchange Monte Carlo method and application to spin glass simulations, Journal of the Physical Society of Japan, 65, 1996, 1604.
[22] R.H. Swendsen and J.-S. Wang, Replica MC simulation of spin-glasses, Physical Review Letters, 57, 1986, 2607.

[23] M.S. Rahman Tito, T.T. Lie, and T. Anderson, Sizing optimization of wind-photovoltaic hybrid energy system under transient load, International Journal of Power and Energy Systems, 33, 2013, 168-174.

[24] A.K. Hartmann and H. Rieger, Optimization algorithms in physics, 1st ed. (Berlin: Wiley-VCH, 2001).

[25] A.K. Hartmann and H. Rieger (eds.), New optimization algorithms in physics (Weinheim: Wiley-VCH, 2004).

[26] P. Kundur, Power system stability and control (New York, NY: McGraw-Hill, 1994).

[27] M. Chandorkar, D. Divan, and R. Adapa, Control of parallel connected inverters in stand-alone AC supply systems, IEEE Transactions on Industry Applications, 29(1), 1993, 136-143.

[28] T. Dewenter, B. Werther, A.K. Hartmann, and H.-P. Beck, Optimierung des dynamischen Verhaltens netzstützender Anlagen am Beispiel der Virtuellen Synchronmaschine, Proc. 13th Symp. Energieinnovation (EnInnov2014), Graz, Austria, 2014.

[29] Y. Chen, R. Hesse, D. Turschner, and H.-P. Beck, Improving the grid power quality using VISMAs, International Conf. on Power Engineering, Energy and Electrical Drives (POWERENG), Malaga, Spain, 2011.

[30] N. Metropolis, A.W. Rosenbluth, M.N. Rosenbluth, A.H. Teller, and E. Teller, Equation of state calculations by fast computing machines, Journal of Chemical Physics, 21, 1953, 1087-1092.

[31] S. Kirkpatrick, C.D. Gelatt, and M.P. Vecchi, Optimization by simulated annealing, Science, 220 (4598), 1983, 671-680.

[32] M. Galassi, J. Davies, J. Theiler, B. Gough, G. Jungman, P. Alken, M. Booth, and F. Rossi, GNU scientific library reference manual, 3rd ed., (Bristol: Network Theory Ltd. 2009).

[33] E. Gabriel, G.E. Fagg, G. Bosilca, T. Angskun, J.J. Dongarra, J.M. Squyres, V. Sahay, P. Kambadur, B. Barrett, A. Lumsdaine, R.H. Castain, D.J. Daniel, R.L. Graham, and T.S. Woodall, Open MPI: Goals, concept, and design of a next generation MPI implementation, Proc. 11th European PVM/MPI Users' Group Meeting, Budapest, Hungary, 2004.

[34] A.K. Hartmann, Big practical guide to computer simulations (Singapore: World Scientific, 2015).

\section{Biographies}

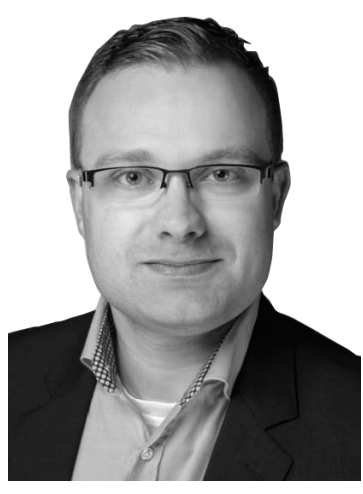

Timo Dewenter was born in Stuttgart, Germany, in 1986. He received his bachelor degree in physics in 2010 from the University of Oldenburg, Germany. In 2012, he obtained his master's degree in physics at the same university, where the topic of the master's degree thesis was disordered magnetic systems. From 2012 to 2016, he has been a Ph.D. student in the working group "Computational Theoretical Physics" at the University of Oldenburg. In the course of his Ph.D. thesis, he worked on convex hulls of multiple random walks, the distribution of resilience of power grids, and the parameter optimization of virtual synchronous machines (in connection with microgrids). Since July 2016, he is working at the Kisters AG in Oldenburg. His research interests are disordered systems, (parameter) optimization, and (simplified) models of power grids also in connection with virtual synchronous machines. 


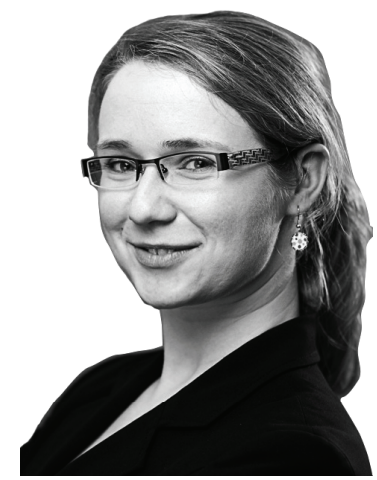

Wiebke Heins was born in Bremervörde, Germany, in 1985. She received her diploma degree in applied mathematics from the Clausthal University of Technology, Clausthal-Zellerfeld, Germany, in 2012. From 2012 to 2015 , she has been a research assistant with the Institute of Electrical Information Technology, Clausthal University of Technology, where she was involved in several research projects on monitoring and control of distribution power systems. Since 2016, she is with the working group for Optimization and Optimal Control at the Center for Industrial Mathematics of the University of Bremen, and the Steinbeis Innovation Center for Optimization and Control, Grasberg, where she is working on optimal scheduling techniques for the control of clustered generation and consumption units. Her research interests include system analysis, optimal scheduling and control of distribution power systems with decentralized and renewable generation.

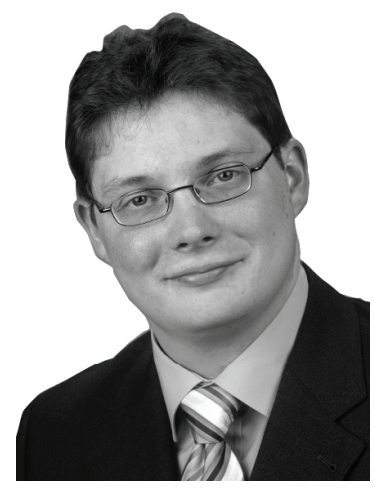

Benjamin Werther was born in Wissen, Germany, in 1977. He received his Diplom-Ingenieur degree in electrical engineering from the Paderborn University, Germany, in 2005. From 2005 to 2009, he was a development and consulting engineer in the company System \& Dynamik in Paderborn. Between 2009 and 2016, he has been a research assistant at the Institute of Electrical Power Engineering and Energy Systems and at the Energy Research Center of Lower Saxony (EFZN), which both are scientific institutions of the Clausthal University of Technology, Clausthal-Zellerfeld, Germany. Since November 2016, he is with the AEG Power Solutions GmbH in Warstein-Belecke. His research interests are in non-linear system theory and electrical power grids.

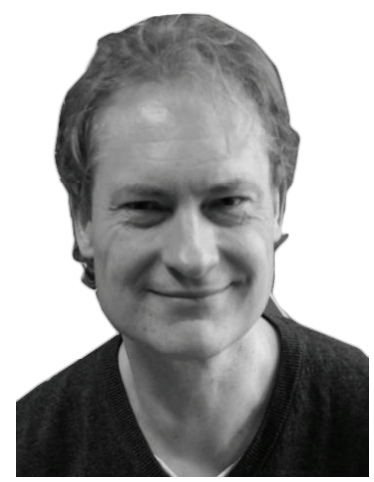

Alexander $K$. Hartmann was born in 1968 in Heidelberg, Germany. From 1987 to 1993, he studied computer science at the FernUniversität in Hagen. He performed his diploma thesis on the simulation of fault-tolerant systems. From 1987 to 1994, he studied physics at the University of Duisburg-Essen, completing with a diploma thesis on the calculation of ground states of diluted antiferromagnets in a field. From 1994, he worked at the University of Heidelberg on simulation of polymer/ noble-gas mixtures and completed his Ph.D. in 1998. He was a postdoctoral at the Universities of Göttingen, Santa
Cruz and Ecole Normale Paris. From 2003, he was heading the junior research group "Complex Ground States of Disorderd Systems" in Göttingen. In 2004, he completed his habilitation. Since 2007, he is Professor for Computational Theoretical Physics at the University of Oldenburg, Gernamy. His research interests are disordered systems, bioinformatics, and optimization problems.

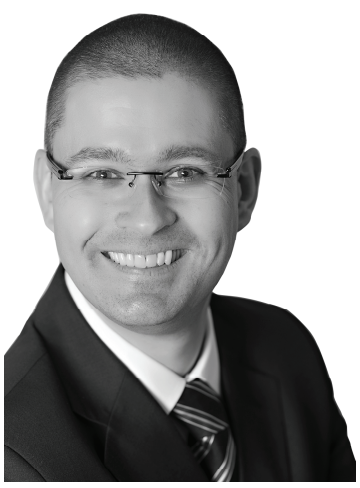

Christian Bohn was born in Hamburg, Germany, in 1969 and studied electrical engineering at the University of Sussex, England, and the Technische Universität Braunschweig, Germany, from which he obtained the DiplomIngenieur degree in 1994. From 1994 to 2000, he was a research associate at the Control Engineering Institute, Ruhr-University Bochum, Germany, where he completed his doctorate in 2000. He then joined Continental AG, where he worked on active noise and vibration control for passenger vehicles. From 2004 to 2007, he was with IAV $\mathrm{GmbH}$, where he worked on control function development for combustion engines and the development of components for hybrid electric vehicles. Since 2007, he has been a professor for control engineering and mechatronics at the Clausthal University of Technology, Clausthal-Zellerfeld, Germany. His research interests are advanced control and estimation methods and their application to industrial systems.

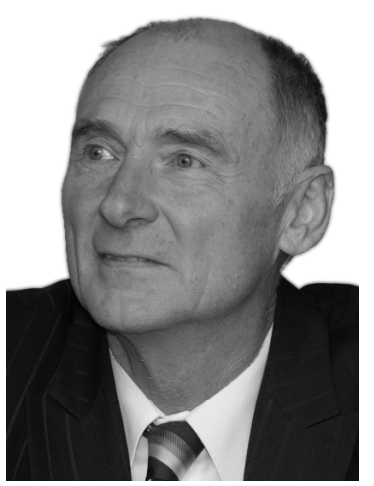

Hans-Peter Beck was born in Ehmen, Germany, in 1947. He received the Diplom-Ingenieur and Ph.D. degree in electrical engineering from the Technische Universität Berlin in 1976 and 1981, respectively. From 1977 to 1989, he was with the Allgemeine Elektricitäts-Gesellschaft (AEG), Berlin, at the branch Electrical Power Engineering. In 1982, he became head of the laboratory Novel Energy Systems, and in 1985 head of the design department Electrical Railway Traction Vehicle of the AEG division Railway Technology. Since 1989, he is professor and director of the Institute of Electrical Power Engineering and Energy Systems, Clausthal University of Technology, Clausthal-Zellerfeld, Germany. Since 2008, he is also managing director of the Energy Research Center of Lower Saxony (EFZN). He is a full member of the Braunschweigische Wissenschaftliche Gesellschaft and of the National Academy of Science and Engineering (acatech), and member of the technical and scientific advisory council of the journal Antriebstechnik. 